\title{
COUPLING BETWEEN FLEXIBLE SHIP AND LIQUID SLOSHING USING POTENTIAL FLOW ANALYSIS
}

\author{
Youngbum Lee \\ Fluid-Structure Interactions Research Group \\ School of Engineering Sciences \\ University of Southampton, Southampton , UK \\ Pandeli Temarel* \\ Fluid-Structure Interactions Research Group \\ School of Engineering Sciences \\ University of Southampton, Southampton , UK
}

\author{
Mingyi Tan \\ Fluid-Structure Interactions Research Group \\ School of Engineering Sciences \\ University of Southampton, Southampton, UK
}

\begin{abstract}
The significant increase in demand for Liquefied Natural Gas (LNG) and the economic aspects of its transportation resulted in increases in the number and size of LNG carriers. One of the design issues for LNG carriers is the sloshing phenomenon because containment systems widely used nowadays have no internal structures. Furthermore, because the weights of ship and cargo are comparable and ship operators want more flexible operations allowing partial fillings in tanks, the coupling effect between ship motions and sloshing requires further investigation, including the effect of ship distortion.
\end{abstract}

The previous study on coupling between rigid body and sloshing shows good agreement between methods of prediction and measurements[1,2]. Hence, in this paper the potential flow approach adopted for the coupling effect between rigid body ship motion and sloshing is extended to flexible ship-partially filled tank system, using the de-singularised Rankine source method. In this case, the global deflection of the flexible ship is used for application of the body boundary condition on the

\author{
Shihua Miao \\ Fluid-Structures Interactions Research Group \\ School of Engineering Sciences \\ University of Southampton, Southampton, UK
}

partially filled tank. The aim of this paper is to investigate the influence of hull flexibility on the hydrodynamic forces and moments associated with liquid sloshing and vice versa, as well as the dynamic characteristics (e.g. resonance frequencies) of the whole system. As there are no experimental results available, the method is validated by comparing hydrodynamic forces from sloshing obtained using rigid and flexible body approaches. The coupling effect between flexible ship and sloshing in partially filled tanks is investigated for an idealized LNG Carrier in beam regular waves, considering different partial filling scenarios.

\section{INTRODUCTION}

As Liquefied Natural Gas (LNG) transportation continues to expand, the demand for building LNG carriers has dramatically increased since early 2000's and this trend is likely to continue for a relatively long period since many countries consider Natural Gas as their future energy supply [3]. One of the design issues for LNG carriers is the sloshing phenomenon because containment systems widely used nowadays have no internal structures. Furthermore, to increase economic profit, there is a huge demand for much larger LNG vessels. As a consequence, 
many investigations have been carried out to predict sloshing loads and the responses of hull and containment system in order to ensure structural integrity of the whole system $[4,5]$.

Amongst the aforementioned topics related to sloshing, studies on interaction effects between ship rigid body motions and sloshing loads are of particular interest. The results are of practical benefit not only for local structural design due to sloshing but also for global ship design. Ship motion performance is considered very critical in ship to ship transfer of liquid cargo and also in some offshore structure (e.g. FPSOs), where a large number of process modules are installed at various deck levels. Distillation tower on deck is another example of operations highly dependent on ship motion.

To ensure a ship's proper performance, motions should be restricted to certain level and several design concepts such as large bilge keel, anti-rolling tanks, etc can be incorporated to solve this problem. Sloshing may produce large forces and moments and consequently affect ship motions. Anti-rolling tanks are good examples of how sloshing affects ship motions [6] and it should be noted that a small amount of control liquid, such as $5 \%$ of total displacement of a ship, could be effectively used to reduce the maximum roll motion at resonance condition [7]. In addition, the demand for partial filling operation inevitably increases the possibility of sloshing problem [8]. However, it should be carefully dealt with because as a result of tuning, roll motion may be larger at other frequency ranges. Thus, good prediction of coupling effects between sloshing loads and ship motions are critical for the design of marine/offshore structures.

This topic is not a new one, but recent strong interest along with enhanced computational capability is the source that enables systematic analysis both in frequency and time domains, as well as experiments. Rognebakke \& Faltinsen [9] carried out non-linear sloshing analysis in time domain and experimentation for two-dimensional problems. Kim [10] and Nam et al [11] investigated the three-dimensional problem in the time domain. The three-dimensional problem was investigated by Malenica et al [12] and Newman [13] in the frequency domain. Molin et al [14] and Nasar et al [15] carried out experiments on a barge with partially-filled tanks.

It is noted that the vast majority of these activities are mainly concerned with coupling between rigid ship motions and sloshing loads. However, due to the fact that larger size ships are on demand and already being built, more effort needs to be focused on the interaction between the ship globally treated as a flexible structure and liquid cargoes. This is because as LNG carrier dimensions increase the overall stiffness of the ship decreases resulting in relatively low distortional resonant frequencies within or close to the range of significant wave energy and excitation. Furthermore this decrease in resonance frequencies associated with distortions results in bringing them closer to sloshing resonance frequencies.

In this paper, as proposed by Malenica et al [12] and Lee et al $[1,2]$, sloshing forces and moments are represented as added mass coefficients. The procedure outlined by Lee et al $[1,2]$ has been validated against analytical and experimental measurements $[9,14,16]$. In this paper this procedure is incorporated into the unified equations of motion of the coupled fluid-flexible structure interaction analysis in frequency domain. As there are no experimental measurements for such a system, the validation of the generalized analysis developed is carried out by comparing with the rigid body case and qualitatively through the investigation of a range of partial filling scenarios for an idealized LNG Carrier stationary in beam waves.

\section{THEORETICAL BACKGROUND \\ 2.1 Sloshing}

A potential flow analysis is used for the coupling between ship rigid motions and liquid sloshing in a tank (or tanks). Previous investigations made use of the pulsating source Green's function $[1,2]$ and the conventional and de-singularised Rankine source approaches [2] for the evaluation of the hydrodynamic actions of liquid inside a partially filled rigid tank (internal problem). The de-singularised Rankine approach is selected, due to its advantages in terms of accuracy and efficiency, for application to liquid inside a partially filled tank subject to the global deformations of a flexible ship. The process is a unified or generalized one, namely it encompasses rigid body motions and distortions. The velocity potential of the Rankine source satisfies Laplace's equation, the linearised free surface condition and body boundary condition shown in (1).

$$
\begin{array}{ll}
\Delta \phi=0 & \text { in the liquid domain } \\
-\frac{\omega^{2}}{g} \phi+\frac{\partial \phi}{\partial z}=0 & \text { on free surface } \\
\frac{\partial \phi_{r}}{\partial n}=i \omega_{e}\left(u_{r} n\right) & \text { on body surface }
\end{array}
$$

where $\varphi_{r}$ denotes the radiation potential ( $\mathrm{r}=1$ surge, $\mathrm{r}=2$ sway, $\mathrm{r}=3$ heave, $\mathrm{r}=4$ roll, $\mathrm{r}=5$ pitch, $\mathrm{r}=6$ yaw, $\mathrm{r}=7,8, \ldots$ distortions), $u_{\mathrm{r}}=\{\mathrm{u}, \mathrm{v}, \mathrm{w}\}_{\mathrm{r}}$ the $\mathrm{rth}$ mode shape, $n$ the normal vector and $\omega_{\mathrm{e}}$ the wave encounter frequency.

In the de-singularised Rankine source method, sources are located outside the liquid domain, but the body and free surface boundary conditions are applied on the actual body surface [2]. This is achieved through the offset $\varepsilon$, which is product of offset factor and square of the area of the four-cornered panels on the walls, bottom and free surface of the tank. Once the velocity potential is obtained the relevant generalized added mass, with 
reference to an axis system based on the free surface of the tank, is obtained as shown by (2), namely

$$
A_{r k}=\left(\rho / \omega_{e}^{2}\right) \operatorname{Re} \iint_{S} n^{T} u_{r}\left(i \omega_{e}\right) \phi_{k} d S
$$

where $\rho$ denotes the density of the liquid in the tanks. A free surface correction when the tank has a vertical movement, as suggested by Malenica et al [12], is extended for the generalized case of the tank in a flexible ship.

For the coupling between flexible body and liquid sloshing, global deflections of a flexible body are used for satisfying the body boundary condition for sloshing analysis. There are several ways of normalization of deformation but a method of generalized unit mass matrix is utilized because it is more convenient when data is transferred from commercial FE codes. An interpolation scheme is used to obtain deflections at tank boundaries from the FE model, allowing for independence between FE model and panel idealization of the free surface and boundaries of the tank.

For a rectangular tank resonances associated with the liquid sloshing inside the tank can be obtained according to Graham \& Rodriguez [16], namely

$\omega_{m n}^{2}=g k_{m n} \tanh \left(k_{m n} h\right)$

where $\mathrm{m}$ and $\mathrm{n}$ denote the modes in longitudinal and transverse directions, respectively, $h$ the depth of liquid inside the tank and $\mathrm{k}_{\mathrm{mn}}$ is given by

$k_{m n}=\pi \sqrt{\frac{(2 m+1)^{2}}{L^{2}}+\frac{(2 n+1)^{2}}{B^{2}}}$

with $\mathrm{L}$ and $\mathrm{B}$ denoting the length and breath of the tank,. For $\mathrm{m}, \mathrm{n}=0,1,2$, etc. It should be noted that for longitudinal modes the second expression is omitted and for transverse modes the first expression is omitted.

\subsection{Generalized equations of motion for flexible ship - partially filled tank system}

The generalized equations of motion in regular waves are

$\left[[a]_{h}+[A]_{h}+[A]_{s l o}\right]\{\ddot{p}\}+\left[[b]_{h}+[B]_{h}\right]\{\dot{p}\}+$ $\left[[c]_{h}+[C]+[C]_{s l o}\right]\{p\}=\{\Xi\} \exp \left(i \omega_{e} t\right)$

This system of equations has the form developed by Bishop et al [17] for fluid-flexible hull interaction. Terms to allow for the effects of sloshing are added, based on the analysis by Malenica et al and Lee et al $[1,2,12]$. In these equations $[a]_{h}$ and $[\mathrm{c}]_{\mathrm{h}}$ denote the hull's generalized mass and stiffness matrices, obtained from dry hull analysis and including the mass of the liquid in the tanks treated as a frozen mass. $[\mathrm{b}]_{\mathrm{h}}$ is the generalized structural damping matrix, assumed diagonal. $[\mathrm{A}]_{\mathrm{h}}$ and $[\mathrm{B}]_{\mathrm{h}}$ denote the encounter frequency dependent generalized added mass and fluid damping of the hull at a draught which accounts for the liquid in the tanks treated as frozen mass. $[\mathrm{C}]_{\mathrm{h}}$ is the corresponding generalized fluid restoring matrix and $\{\Xi\}$ the wave excitation vector, comprising incident wave and diffraction components. $[\mathrm{A}]_{\mathrm{h}}$, $[B]_{h}$ and $\{\Xi\}$ were evaluated using the three-dimensional hydroelasticity theory developed by Bishop et al [17], using a distribution of pulsating sources over the mean wetted surface of the hull. In previous investigations, fluid damping was augmented to account for viscous effects in way of roll motion, in order to get more meaningful comparisons with experimental measurements [2]. These effects are not included in this paper.

$[\mathrm{A}]_{\text {slo }}$ denotes the frequency dependent generalized added mass matrix due to the liquid in the tanks, suitably transformed to the axis system used for ship motions and distortions. Lee et al discussed the trends of these added mass values as the frequency approaches zero $[1,2]$. Allowance has to be made so that the "zero frequency" values, e.g. the frozen liquid mass and inertia, are not accounted twice. This allowance is extended to all modes, under the assumption that as the frequency tends to zero there should be no influence due to the liquid in the tanks. These effects are included when using the generalized added masses of (2) to generate the $[\mathrm{A}]_{\text {slo }}$ matrix. It should be noted that $[\mathrm{C}]_{\text {slo }}$ only contains corrections associated with rigid body modes, allowing for the influence of free surface on the roll and pitch restoring moments. The necessity for a correction to distortion modes is still under consideration.

\section{APPLICATION TO AN IDEALIZED LNG CARRIER}

The aforementioned analysis is applied to an idealized LNG carrier stationery in beam regular waves, considering various scenarios with partially filled tanks. The principal particulars of the LNG carrier are given in Table 1 . They correspond to a real LNG Carrier, of $200000 \mathrm{~m}^{3}$ capacity, and of same length, breadth, depth and displacement. However, for simplicity of application the shape of the hull is rectangular. It is divided into 5 tanks of $63 \mathrm{~m}$ length each, as shown in Figure 1. Three scenarios of partially filled tanks are considered: (a) Tank 3 alone, (b) Tank 1 alone and (c) all tanks, acting as one tank for the whole ship. It should be noted that for all the scenarios with partially filled tanks considered the total displacement of the idealized LNG carrier is maintained at a constant value of 142500 tonnes. For case (c) the LNG mass is 124740 tonnes, all treated as liquid. For cases (a) and (b) the liquid LNG mass in partially filled tank is 24948 tonnes, with the remainder of the LNG mass treated as frozen. Although unrealistic, this was done in order to perform comparison of the response without having to account for variations in total mass and inertia. Furthermore, for simplicity of analysis, only one FE structural model was constructed and employed for all partially filled 
tank scenarios, using uniform mass distribution, including the liquid in the tanks treated as frozen.

\subsection{Dry and wet hull analyses of idealized LNG, excluding influence of sloshing}

The FE model of the idealized LNG carrier is shown in Figure 1. It has double skin on the sides, but only single bottom. The FE model shown in Figure 1 does not contain the "fictitious bulkheads" of negligible material density used, as per past practice [18], in order to eliminate local deformations appearing in the global mode shapes. These account for structural detail such as common frames and web frames which are not included in the FE model. The deck plating in the idealized LNG carrier is not shown, to enable internal view. The FE model comprises 6522 shell elements in total).

The dry hull or in vacuo natural frequencies for the first 5 modes are shown in Table 2. These are of the same order of magnitude as those estimated for the real ship. This set of mode shapes, comprising two vertical bending, two horizontal bending and one twisting mode, is ideal for illustrating the application for both symmetric and antisymmetric dynamic behavior, though the latter is much more important in the beam regular waves investigated.

A selection of generalized added masses, $\mathrm{A}_{22}$ (sway), $\mathrm{A}_{44}$ (roll), $\mathrm{A}_{77}$ (2-node vertical bending), $\mathrm{A}_{88}$ (two-node horizontal bending) and $\mathrm{A}_{99}$ (one-node twisting), for the idealized $\mathrm{LNG}$ carrier oscillating in water, i.e. liquid mass in tanks treated as frozen, are shown in Figure 2. These were obtained using a relatively crude 338 panel idealization of the mean wetted surface. These can be useful in comparing the magnitudes of the generalized added masses due to liquid in partially filled tank(s). The principal coordinate amplitudes of the LNG carrier, liquid mass in tanks treated as frozen (or uncoupled case) will be discussed later and are shown in Figure 5.

\subsection{Evaluation of sloshing hydrodynamic properties}

A selection of generalized added masses due to partially filled tank 3 alone (the tank in the middle) are shown in Figure 3. Tank 3 is filled to depth $\mathrm{h}=20 \mathrm{~m}$. Please note that the added mass values in Figures 3 and 4 are for density $\rho=1000 \mathrm{~kg} / \mathrm{m}^{3}$. All added masses are with reference to the equilibrium axis system of the hull, located at the LCG. When these are incorporated into (4) they are modified to account for LNG density $\rho=450 \mathrm{~kg} / \mathrm{m}^{3}$. All remaining tanks are assumed to contain liquid treated as frozen mass. This is an extreme, and probably unpractical, case used to illustrate the working of the methodology. Generalized added masses, due to sloshing, $\mathrm{A}_{22}$ and $\mathrm{A}_{44}$ evaluated using the current unified approach agree with those obtained using the previous rigid body approach by Lee et al [2], as shown in Figure 3. This is the case for all rigid body elements of $[\mathrm{A}]_{\text {slo, }}$, indication the accuracy of the numerical methods employed.
The natural frequencies associated with liquid sloshing in Tank 3 (or 1), evaluated using (3) are shown in Table 3. Their influence can be seen in the generalized added masses of Figure 3 , for example the $1^{\text {st }}$ and $2^{\text {nd }}$ transverse modes in $\mathrm{A}_{22}$ and $\mathrm{A}_{88}$, the $1^{\text {st }}$ transverse mode in $\mathrm{A}_{44}$, the $1^{\text {st }}$ diagonal mode in $\mathrm{A}_{99}$ etc.

Comparing Figures 2 and 3 one can see that, in general, the magnitudes of the generalized masses are of same order. A notable exception may be the large magnitude of the peak for the sway generalized added mass due to liquid sloshing.

Understanding the interaction between hull motions and distortions, i.e. the mode shapes and direction of sloshing loads is important in explaining the extent of coupling. For example, symmetric modes (e.g. two-node VB) will not result in significant sloshing loads in the longitudinal direction, even when considering the case of Tank 1 partially filled alone. This will result in weak coupling between the elements of the symmetric mode shapes of $[\mathrm{A}]_{\text {slo. }}$.

On the other hand, this is not the case for the antisymmetric modes, as illustrated in Figure 4. Comparing cases (a) and (c) (namely, tank 3 alone and whole ship partially filled, respectively) one notes that case (c) results in much larger values for $A_{22}$, especially at resonance, as one would physically expect. Furthermore, the partial filling scenario also affects the coupling. For example in case (c) the distribution of the sway force is such that it decreases the influence of the two-node horizontal bending mode, whilst for case (a) this influence is more pronounced. This can be clearly seeing from the values of $\mathrm{A}_{82}$, shown in Figure 4. A similar reasoning also applies to the coupling between modes $r=4$ (roll) and $r=8$, shown for $A_{84}$ in Figure 4 . These and other examples, not included in the paper, show that the method adopted provides results which are qualitatively right.

\subsection{Wet hull analysis of ideliased LNG, including influence of sloshing}

The amplitudes of the principal coordinates of the idealized LNG carrier stationary in beam regular waves, for partial filling scenarios (a), (b) and (c), as well as for the case where the liquid is treated as frozen, denoted as "uncoupled" are shown in Figure 5. These are evaluated from (4), using the appropriate values for the matrices. Only potential flow damping is used for the external fluid, resulting in relatively large magnitudes for $\mathrm{p}_{4}$ at resonance for the "uncoupled" case. The influence due to sloshing in partially filled tanks can be seen in $p_{4}$ for all cases (a), (b) and (c) in the form of two resonant peaks, one lower and one higher than the roll resonance for the uncoupled case. This is very clear for case (c), i.e. whole ship partially filled, with two peaks of small magnitude around 0.5 and $1 \mathrm{rad} / \mathrm{s}$. In cases (a) and (b), tanks 3 and 1 partially filled respectively, there is negligible difference between them for the first peak, of same magnitude as the "uncoupled" case. Furthermore, the 
second peak is rather small for either case. This is attributed to the relatively small amount of liquid mass for these two scenarios. Similar observations can be made for the sway principal coordinate amplitude $\mathrm{p}_{2}$, shown in Figure 5. All three scenarios result in a trough around $0.75 \mathrm{rad} / \mathrm{s}$, attributable to the $1^{\text {st }}$ transverse sloshing resonance (see Table 3 ) and a peak around $1 \mathrm{rad} / \mathrm{s}$, with case (c) showing the largest variations. Furthermore, for case (c) a second peak is also noted which can be attributed to the $2^{\text {nd }}$ transverse sloshing resonance at 1.45 $\mathrm{rad} / \mathrm{s}$ (see Table 3).

For the symmetric motions, heave, as expected, will result in negligible differences between all cases. This is also true for the symmetric distortional principal coordinate amplitudes. For example one notes that for $\mathrm{p}_{7}$, in Figure 5 , there is very little difference between all three partial filling scenarios and the uncoupled case up to about $2 \mathrm{rad} / \mathrm{s}$. The wet resonance frequency associated with the two-node VB distortion is around $2.5 \mathrm{rad} / \mathrm{s}$. In this vicinity there appear to be some differences between the various scenarios. Whilst the uncoupled case and case (b), tank 3 alone, show sharp peaks at this wet resonance, the peaks appear much smaller for cases (a) and (c). This aspect may require further investigation, as it may be important in terms of springing and whipping.

For the antisymmetric distortions the amplitude for $\mathrm{p}_{8}$, the twonode HB mode, is 20 times larger for cases (a) and (b) in the vicinity of $1 \mathrm{rad} / \mathrm{s}$ compared to the uncoupled case. This is a result of significant coupling between sway (mode $\mathrm{r}=2$ ) and roll (mode $r=4$ ) and mode $r=8$, which was discussed in section 3.2. The consequences will be that the resultant horizontal bending moment will have much larger values for partially filled cases similar to cases (a) and (b) compared to the uncoupled case. This aspect may require further verification in terms of additional scenarios, even though normal operational conditions do not allow for one tank to be partially filled in beam seas. For case (c), i.e. whole ship partially filled the magnitudes of $p_{8}$ are of similar order as those of the uncoupled case, though the occurrence of peaks is influenced by sloshing resonances. All cases behave in a similar manner in the vicinity of the wet resonance frequencies associated with modes $r=8$ and $\mathrm{r}=11$.

The amplitude of the first twisting mode $\mathrm{p}_{9}$, follows by and large the trends seen in $p_{4}$. That is to say there is very little difference between cases (a) and (b) in the vicinity of the first peak, which is slightly larger than that of the uncoupled case, as in $\mathrm{p}_{4}$. However, the difference between cases (a) and (b) around $1 \mathrm{rad} / \mathrm{s}$ is pronounced, with case (b) displaying a substantial peak. This is attributed to the more significant contributions from mode $r=9$, one-node twisting, for the case of tank 1. Case (c) has the smallest peak values, similar to $\mathrm{p}_{4}$. The behavior of all cases is fairly similar in the vicinity of the wet resonance frequencies associated with antisymmetric distortions.

\section{CONCLUSIONS}

A method for evaluating the interactions between liquid sloshing in partially filled tanks and hull motions and distortions has been developed and illustrated, for both symmetric and antisymmetric hull distortions, using an idealized LNG Carrier stationary in beam waves and various partial filling scenarios.

The method is based on evaluating the added mass contributions due to liquid in partially filled tanks, using potential flow analysis and the Rankine de-singularised approach. The unified procedure was validated by comparing added mass coefficients for rigid body modes, with those obtained previously using only a rigid body analysis $[1,2]$. This limited validation, provides confidence on the accuracy of the numerical method. The added mass coefficients associated with distortional modes, and their coupling to rigid body modes, were qualitatively assessed using three different partial filling scenarios. The indications are that the method developed provides predictions whose trends can be explained physically.

Further work should focus on more partial filling scenarios to confirm the behavior of some distortional responses. A more realistic LNG carrier, with realistic filling scenarios and operations conditions, i.e. heading and forward speed should also be investigated.

\section{ACKNOWLEDGEMENT}

The authors would like to thank Daewoo Shipbuilding \& Marine Engineering, Co. Ltd. for their kind support.

\section{REFERENCES}

[1] Lee, Y., Tan, M. and, Temarel, P., 2008, "Coupling between ship motion and sloshing using potential flow analysis", International Conference on Hydrodynamics.

[2] Lee,Y., Godderidge, B., Tan, M., Temarel, P., Turnock, S. and Cowlan, N., 2009, "Coupling between ship motion and sloshing using potential flow analysis and rapid sloshing model", Proceedings of the nineteenth International Offshore and Polar Engineering Conference, Osaka, Japan, Vol. 1, pp. 54-62.

[3] Sember, W.J., 2004, "Demand for natural gas: the role class plays in LNG transportation", Proceedings of the $14^{\text {th }}$ Offshore Symposium, Houston, Texas, Texas section of the Society of Naval Architects and Marine Engineers.

[4] Zhao, R. and Rognebakke,.O., 2004, "Global and local loads in design of large LNG ships", Proceedings of the $14^{\text {th }}$ Offshore Symposium, Houston, Texas, Texas section of the Society of Naval Architects and Marine Engineers.

[5] Kim, J.W., Lee, H. and Shin, Y., "Sloshing impact load and strength assessment of membrane-type LNG containment system in large LNG carriers", Proceedings of the $14^{\text {th }}$ Offshore Symposium, Houston, Texas, Texas section of the Society of Naval Architects and Marine Engineers. 
[6] Kim, Y., 2002, "A numerical study on sloshing flows coupled with ship motion-The anti-rolling tank problem", Journal of Ship Research, Vol.46, No.1:52-62.

[7] Lloyd, A.R.J.M., 1998, Ship behaviour in rough weather, Ellis Horwood.

[8] Zalar, M., Cambos, P., Gallo, B. and Mravak, Z., 2005, "Partial filling of membrane type LNG carriers", GASTECH.

[9] Rognebakke, O.F. and Faltinsen, O.M., 2001, "Effect of sloshing on ship motions", 16th International Workshop on Water Waves and Floating Bodies (IWWWFB), Japan.

[10] Kim, Y., 2001, "Coupled analysis of ship motions and sloshing flows", 16th International Workshop on Water Waves and Floating Bodies (IWWWFB), Japan.

[11] Nam, B., Kim, Y. and Kim, D., 2006, "Nonlinear Effects of Sloshing Flows on Ship Motion", 21st International Workshop on Water Waves and Floating Bodies (IWWWFB), UK.

[12] Malenica, S., Zalar, M. and Chen, X.B., 2003, "Dynamic coupling of seakeeping and sloshing", 14th Int. Offshore and Polar Eng. Conf., Honolulu.

[13] Newman, J.N. 2005. "Wave effects on vessels with internal tanks", 20th International Workshop on Water Waves and Floating Bodies (IWWWFB), Spitsbergen. Norway.

[14] Molin, B., Remy, F., Rigaud, S. and de Jouette, C., 2002, "LNG-FPSOs: frequency domain, coupled analysis of support and liquid cargo motions", IMAM Greece.

[15] Nasar, T., Sannas, S.A. and Sundar, V., 2008, "Experimental study of liquid sloshing dynamics in a barge carrying tank", Journal of Fluid Dynamics Research, Vol.40: 427-458.

[16] Graham, E.W. and Rodriguez, A.M., 1952, "The characteristics of fuel motion which affect airplane dynamics", Journal of Applied Mechanics, Vol.19, No.3, 381-388.

[17] Bishop, R.E.D., Price, W. G., and Wu, Y., 1986, “A general linear hydroelasticity theory of floating structures moving in a seaway", Phil. Trans. R. Soc. Lond., A 316, pp.375-426.

[18] Hirdaris, S.E., Price, W.G. and Temarel, P., 2003, "Twoand three-dimensional hydroelastic modeling of a bulker in regular waves", Marine Structures, 16, 627-658.

Table 1. Main particulars of idealized LNG Carrier.

\begin{tabular}{|l|c|}
\hline \hline Ship length (m) & 315 \\
\hline Ship breadth (m) & 50 \\
\hline Ship depth (m) & 27 \\
\hline Ship draft (m) & 8.8 \\
\hline Tank breadth (m) & 44 \\
\hline Displacement (tonnes) & 142,500 \\
\hline
\end{tabular}

* Author of correspondence
Table 2. Dry hull natural frequencies $\omega_{\mathrm{r}}(\mathrm{Hz})$ of idealized LNG Carrier; VB: Vertical bending, HB: Horizontal bending, T: Twisting.

\begin{tabular}{|l|c|}
\hline Mode & $\omega_{\mathrm{r}}$ \\
\hline $\mathrm{r}=7(2$ node $\mathrm{VB})$ & 0.55 \\
\hline $\mathrm{r}=8(2$ node $\mathrm{HB})$ & 0.91 \\
\hline $\mathrm{r}=9(1$ node $\mathrm{T})$ & 1.12 \\
\hline $\mathrm{r}=10(3$ node $\mathrm{VB})$ & 1.32 \\
\hline $\mathrm{r}=11(3$ node $\mathrm{HB})$ & 1.91 \\
\hline
\end{tabular}

Table3. Sloshing natural frequencies (rad/s) for Tank 1 (or 3 ) and the whole ship filled with liquid, based on (3).

\begin{tabular}{|l|c|c|}
\hline \multicolumn{1}{|c|}{ Mode } & $\begin{array}{c}\text { Tank 1 (or 3) } \\
\mathrm{h}=20 \mathrm{~m}\end{array}$ & $\begin{array}{c}\text { Whole Ship } \\
\mathrm{h}=20 \mathrm{~m}\end{array}$ \\
\hline Longitudinal $(\mathrm{m}=0)$ & 0.61 & 0.14 \\
\hline Longitudinal $(\mathrm{m}=1)$ & 1.21 & 0.4 \\
\hline Longitudinal $(\mathrm{m}=2)$ & 1.56 & 0.61 \\
\hline Transverse $(\mathrm{n}=0)$ & 0.79 & 0.79 \\
\hline Transverse $(\mathrm{n}=1)$ & 1.45 & 1.45 \\
\hline Transverse $(\mathrm{n}=2)$ & 1.87 & 1.87 \\
\hline Diagonal $(\mathrm{m}=0, \mathrm{n}=0)$ & 0.90 & 0.8 \\
\hline Diagonal $(\mathrm{m}=1, \mathrm{n}=0)$ & 1.27 & 0.83 \\
\hline Diagonal $(\mathrm{m}=0, \mathrm{n}=1)$ & 1.47 & 1.45 \\
\hline
\end{tabular}

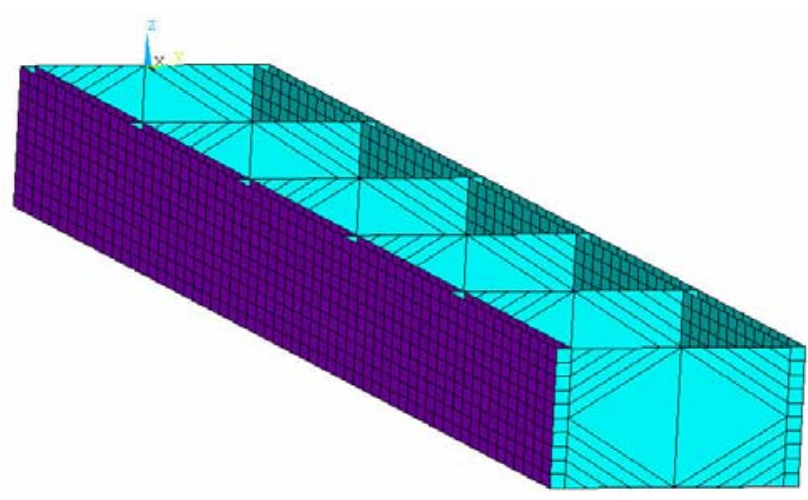

Figure1. FE model of the idealized LNG Carrier; axis system at $A P$, tanks numbered as $5,4,3,2$ and 1 from AP. 

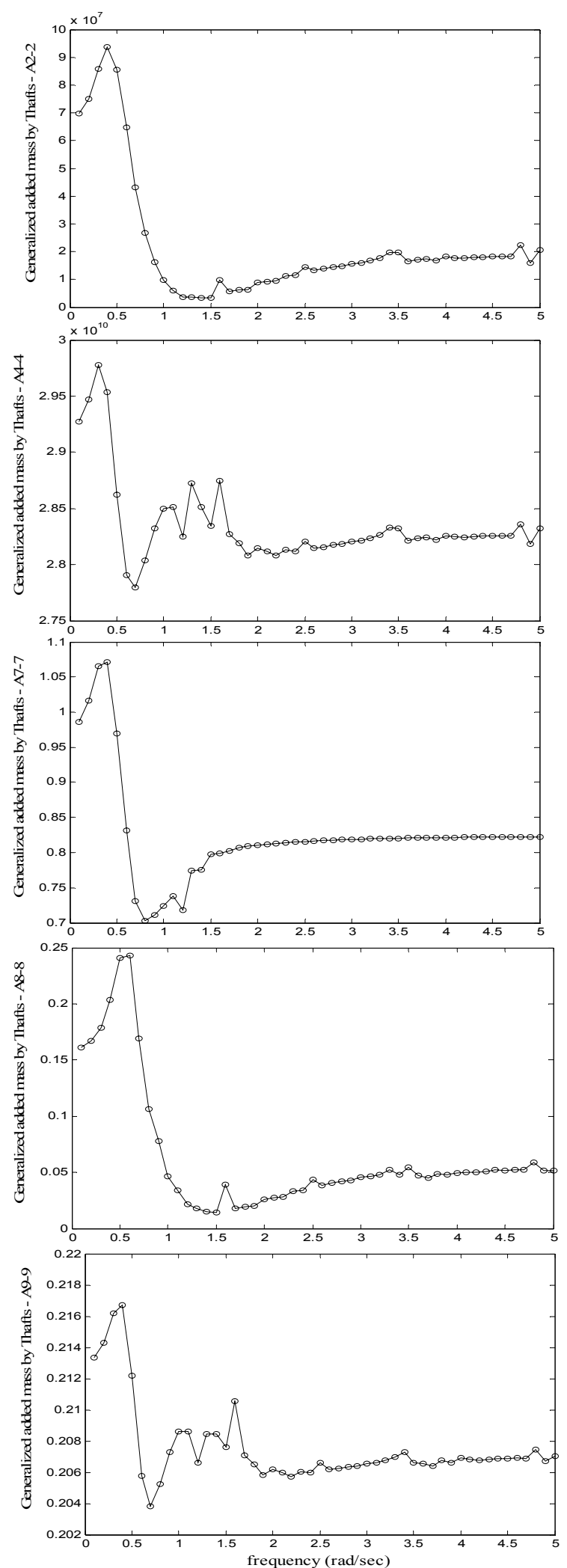

Figure 2. Selection of generalized added mass variations with frequency for the LNG Carrier oscillating in water (uncoupled problem).
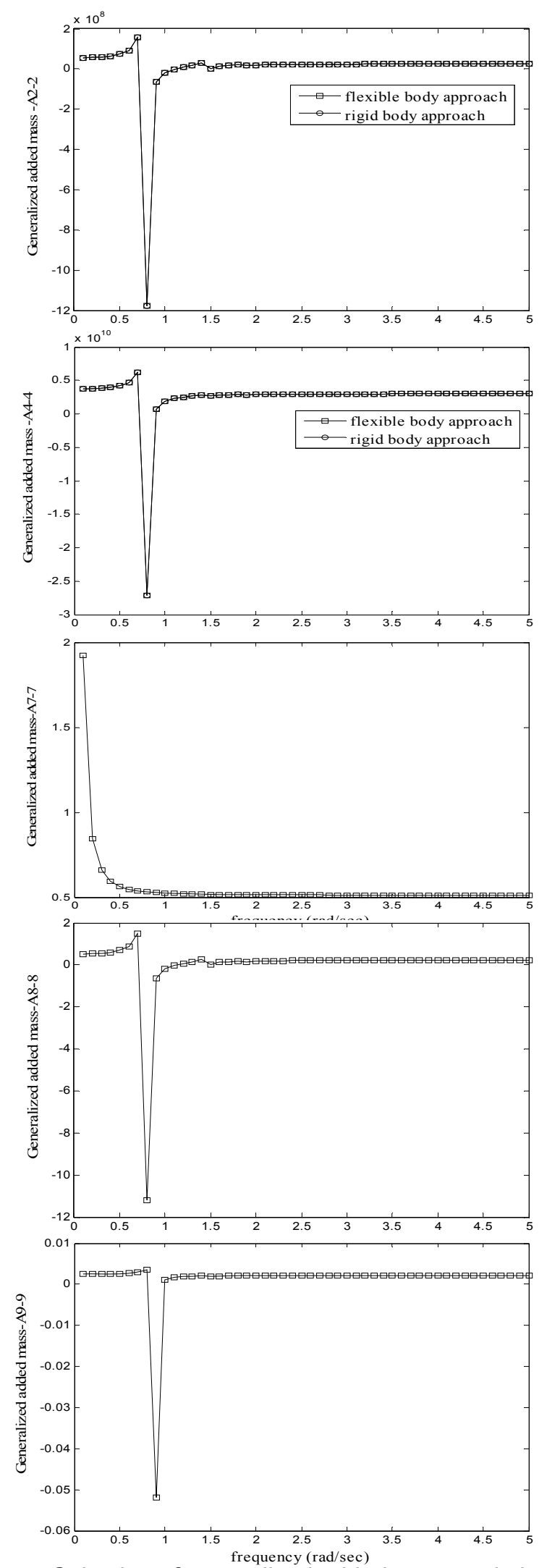

Figure 3. Selection of generalized added mass variations with frequency due to liquid in partially filled tank 3. 

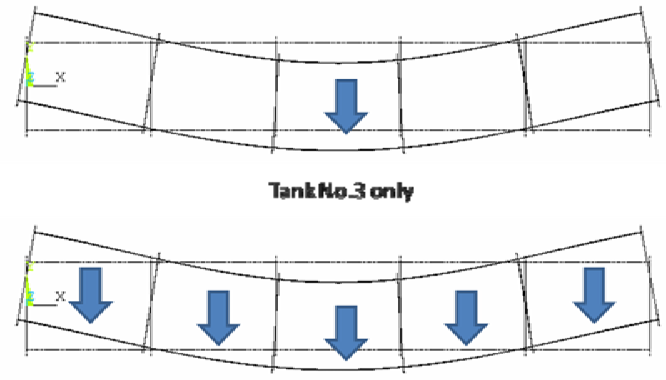

Wholeship liquid without compartments
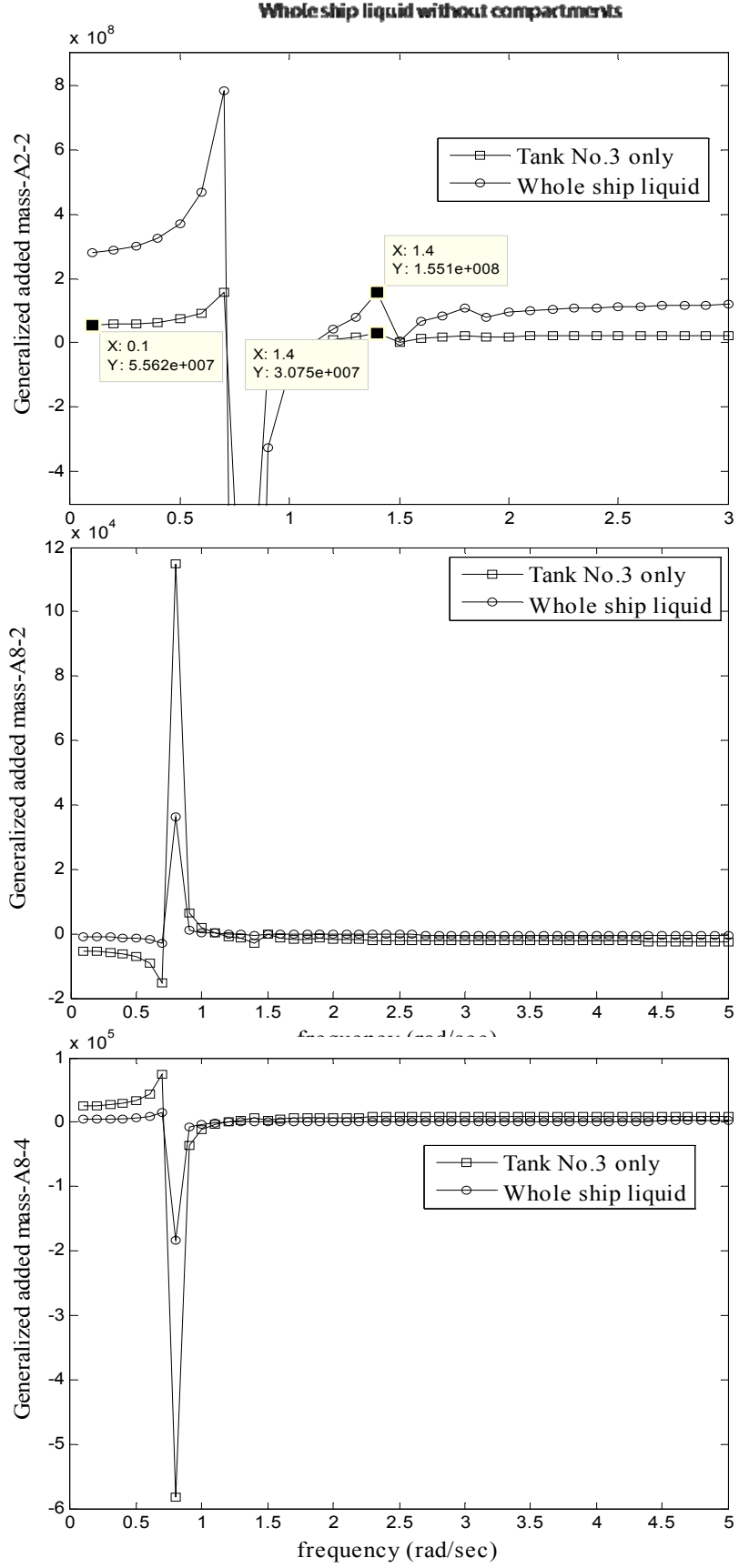

Figure 4. Illustration of tank position and coupling on generalized added mass for partially filled tanks.
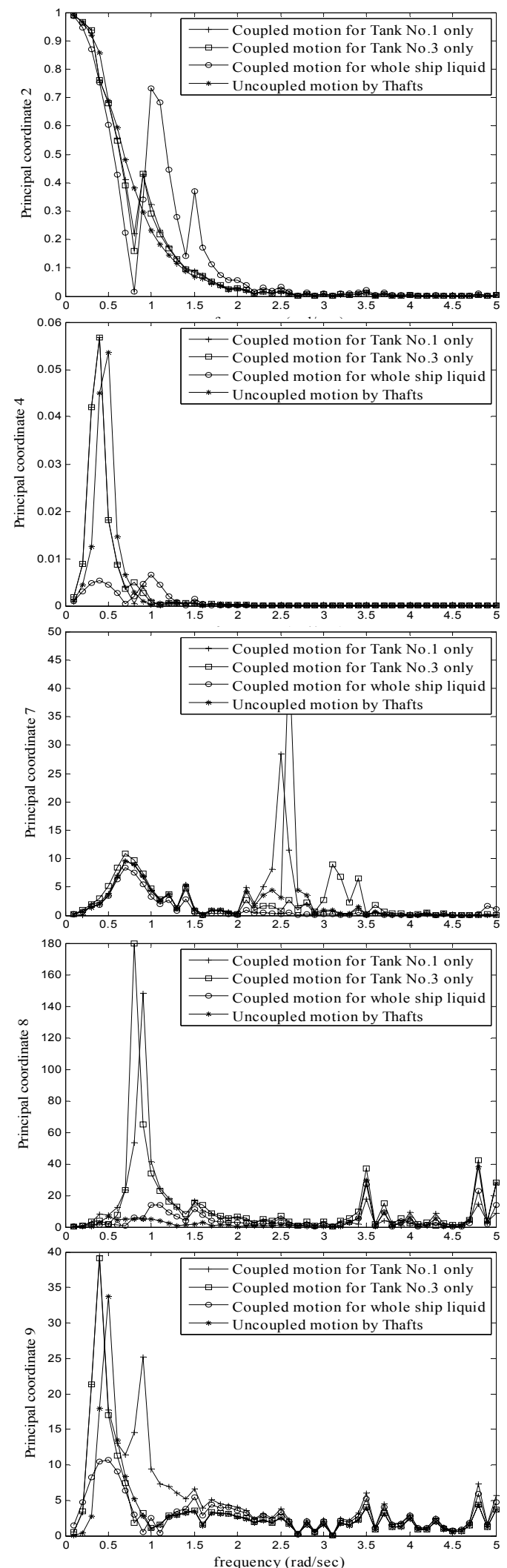

Figure 5. Variation of principal coordinates for the LNG Carrier in beam waves, illustrating the influence of liquid sloshing with different configurations. 